\title{
Ultrasound guided percutaneous cutting biopsy for the diagnosis of pulmonary consolidations of unknown aetiology
}

\author{
Pan-Chyr Yang, Dun-Bing Chang, Chong-Jen Yu, Yung-Chie Lee, Sow-Hsong Kuo, \\ Kwen-Tay Luh
}

\begin{abstract}
Background Ultrasound has been used to guide percutaneous aspiration biopsy of thoracic tumours with high diagnostic yield. This study assessed the diagnostic value of ultrasound guided percutaneous cutting biopsy for pulmonary consolidation of unknown aetiology.

Methods Thirty patients with undiagnosed lobar or segmental consolidation underwent ultrasound guided percutaneous needle aspiration and large bore cutting biopsy. The needle aspirates and biopsy specimens were sent for cytological, microbiological, and histopathological examination.

Results Percutaneous needle aspiration provided a diagnosis in nine of $\mathbf{3 0}$ patients $(30 \%)$, whereas cutting biopsy obtained a satisfactory specimen for histological diagnosis in 28 patients $(93 \%)$ and provided a definite aetiological diagnosis in 17 patients $(57 \%)$. The combination of needle aspiration with Trucut biopsy provided a diagnostic rate of $63 \%$. The underlying diagnoses were fungal pneumonia (five patients), tuberculosis (five), bacterial pneumonia (one), bronchioloalveolar carcinoma (three), lymphoma (two), adenocarcinoma (one), vasculitis (one), acute pneumonia of unknown aetiology (one), and chronic non-specific pneumonia (nine). Two patients, who had necrotic tissue only in the biopsy specimen, were found at thoracotomy to have an adenocarcinoma and aspergillosis. Two patients had complications from the technique, a small pneumothorax in one and a small haemoptysis in another.

Conclusions Ultrasound guided percutaneous cutting biopsy is a valuable method for diagnosing pulmonary consolidation of unknown aetiology. The diagnostic yield is high and the procedure appears to be relatively safe.
\end{abstract}

Pulmonary consolidation has many causes. ${ }^{1}$ Although pneumonia is the most common, non-infectious diseases such as lymphoma, infarction, bronchioloalveolar carcinoma and vasculitis may also present as pulmonary consolidation. For patients with consolidation unresponsive to antibiotics sputum examina- tion, serological tests, and fibreoptic bronchoscopy with biopsy are rational diagnostic approaches. If these procedures fail, the patient may require a more invasive procedure, such as percutaneous needle aspiration or lung biopsy, to determine the diagnosis. Most percutaneous aspirations for pulmonary consolidation have been carried out through a fine needle $\mathrm{e}^{2-6}$ and the material aspirated is often too small to allow detailed microbiological or histological study. Although diagnostic yields of $31-77 \%$ have been reported for infections, the procedure is not sensitive for non-infectious lesions ${ }^{4-6}$ and some patients will require a thoracotomy for a definite diagnosis.

Ultrasound has been used recently to guide percutaneous aspiration biopsy of thoracic lesions. ${ }^{7-9}$ The high quality of image resolution and the precise guiding system have made the percutaneous puncture a safe procedure with a high diagnostic yield..$^{811}$

In this study we have assessed whether ultrasound guided percutaneous cutting biopsy can be used to obtain a diagnosis in patients with pulmonary consolidation not diagnosed by conventional methods, such as fibreoptic bronchoscopy with biopsy. We compare the diagnostic yield of large bore cutting biopsy with that obtained by fine needle aspiration, and discuss the technical aspects and complications of the procedure.

\section{Methods}

Thirty patients with undiagnosed lobar or segmental consolidations underwent transthoracic fine needle aspiration and large bore cutting biopsy under ultrasound guidance from January 1986 to December 1990 . The criteria for selecting patients were: (1) radiographic evidence of lobar or segmental consolidations that extended to the visceral pleura; (2) treatment with antibiotics for at least a week without improvement; (3) failure to make a diagnosis by conventional methods, including sputum examination and fibreoptic bronchoscopy with biopsy. Thirty patients were included in the study, 23 men and seven women aged 20-69 (mean 56) years. Six had underlying malignancy (two leukaemia, one lymphoma, and three a solid tumour) treated previously with chemotherapy. Four patients had diabetes and three had a collagen disease (two systemic lupus erythematosus and one mixed connective 
tissue disease); one had rheumatic heart disease. The other 16 patients had no known underlying disease. Twenty six patients had respiratory symptoms of cough, fever, and sputum production. Two patients had been given amphotericin $B$ in addition to antibiotics. Consolidation occurred in the right upper lobe in 15 patients, the middle lobe in two, the right lower lobe in four, the left upper lobe in four, and the left lower lobe in one; four had more than one affected lobe.

Patients were screened for coagulation abnormalities before the biopsy and excluded if they had thrombocytopenia platelets $(<1.0 \times$ $\left.10^{10} / 1\right)$ or prolonged prothrombin time (prolonged by over three seconds). All patients were examined with real time, linear array, convex, and sector ultrasonic units with a 3.5 , 3.75 and 5.0 MHz transducer (Aloka SSD 630, Toshiba 100A, Tokyo). The sector ultrasonic units were also integrated with a Doppler ultrasound to identify vascular structures. The patients were scanned in the supine posture by an intercostal approach. The sonographic images were recorded on Polaroid films (Polaroid, Cambridge, Massachusetts) and the area of consolidation, the pleural line, and the air and fluid bronchograms were identified. The echogenicities of liver and gall bladder were used as reference echoes for solid and liquid lesions. After the ultrasound assessment the patients underwent percutaneous needle aspiration with a 22 gauge needle and a large bore cutting biopsy with a 16 gauge Trucut needle (Top Surgical, Tokyo). The technique of ultrasound guided percutaneous needle aspiration and cutting biopsy is described elsewhere. ${ }^{8-10}$ After the consolidated lesons had been assessed by ultrasound the skin was prepared and a local anaesthetic agent applied. A sterile puncture transducer (Aloka UST-507 BP) with a preset puncture area and a guiding channel was used to localise the lesion. The aspiration needle or Trucut needle was inserted through a guiding channel and advanced to the consolidated lung parenchyma under real time image monitoring and aspiration or biopsy was conducted. The biopsy route was chosen to avoid penetrating areated lung, major bronchi, or vessels. Patients were asked to hold their breath during the biopsy procedure, which usually took 10 seconds.

Patients usually received two or more needle aspirations followed by a cutting biopsy. The aspirated materials were sent for cytological examination and microbiological cultures. The tissue specimens obtained by cutting biopsy were divided and sent for histopathology and microbiological cultures. Gram staining, acid fast staining, and bacterial and fungal cultures were included routinely. After the aspiration biopsy the patient was observed for one hour and a routine chest radiograph was taken the next day to assess any potential complications.

\section{Results}

Twenty four patients had two percutaneous needle aspirations and six patients had three

Results of ultrasound guided needle aspiration and cutting biopsy in 30 patients with pulmonary consolidation

\begin{tabular}{|c|c|c|c|c|c|}
\hline Patient no & Age (y) & Sex & Location of lesion & Needle aspiration & Cutting biopsy \\
\hline 1 & 40 & $\mathbf{F}$ & RLL & Necrotic tissue & Aspergillosis \\
\hline 2 & 27 & $\mathbf{F}$ & RUL & Necrotic tissue & Aspergillosis \\
\hline 3 & 63 & $\mathbf{F}$ & RUL & Aspergillosis & Aspergillosis \\
\hline 4 & 64 & $\mathbf{M}$ & RUL & Negative & Candidiasis \\
\hline 5 & 69 & $\mathbf{M}$ & LUL & Negative & Pseudodallescheria boydii \\
\hline 6 & 65 & $\mathbf{M}$ & RML & $\begin{array}{l}\text { Actinobacillus } \\
\text { actinomycetemcomitans }\end{array}$ & Acute pneumonia \\
\hline 7 & 20 & $\mathbf{M}$ & RML & Negative & Acute pneumonia \\
\hline 8 & 38 & $\mathbf{M}$ & RUL & Negative & Tuberculosis \\
\hline 9 & 78 & $\mathbf{M}$ & RUL & Tuberculosis & Tuberculosis \\
\hline 10 & 74 & $\mathbf{M}$ & RUL & Negative & Tuberculosis \\
\hline 11 & 64 & $\mathbf{M}$ & RUL & Tuberculosis & Tuberculosis \\
\hline 12 & 66 & $\mathbf{F}$ & RUL & Tuberculosis & Tuberculosis \\
\hline 13 & 39 & $\mathbf{M}$ & LUL & Negative & Chronic inflammation \\
\hline 14 & 66 & $\mathbf{M}$ & RUL & Negative & Chronic inflammation \\
\hline 15 & 61 & $\mathbf{M}$ & RLL & Negative & Chronic inflammation \\
\hline 16 & 71 & $\mathbf{M}$ & RUL & Negative & Chronic inflammation \\
\hline 17 & 47 & $\mathbf{M}$ & RUL & Suspicious & Chronic inflammation \\
\hline 18 & 30 & $\mathbf{M}$ & RUL & Negative & Chronic inflammation \\
\hline 19 & 66 & $\mathbf{M}$ & RUL & Negative & $\begin{array}{l}\text { Chronic inflammation with } \\
\text { fibrosis }\end{array}$ \\
\hline 20 & 67 & $\mathbf{M}$ & RLL & Negative & $\begin{array}{l}\text { Chronic inflammation with } \\
\text { fibrosis }\end{array}$ \\
\hline 21 & 67 & $\mathbf{M}$ & RUL & Negative & $\begin{array}{l}\text { Chronic inflammation with } \\
\text { fibrosis }\end{array}$ \\
\hline 22 & 27 & $\mathbf{M}$ & RML + LUL & Negative & Vasculitis \\
\hline 23 & 63 & $\mathbf{M}$ & RUL & Lymphoma & Lymphoma \\
\hline 24 & 51 & $\mathbf{M}$ & RML + LLL & Lymphoid hyperplasia & Lymphoma \\
\hline 25 & 67 & $\mathbf{M}$ & RML + RUL & Negative & Bronchioloalveolar ca \\
\hline 26 & 38 & $\mathbf{M}$ & LLL & Bronchioloalveolar ca & Bronchioloalveolar ca \\
\hline 27 & 60 & $\mathbf{M}$ & RLL & Bronchioloalveolar ca & Bronchioloalveolar ca \\
\hline 28 & 55 & $\mathbf{F}$ & RML + LUL & Negative & Adenocarcinoma \\
\hline 29 & 66 & $\mathbf{M}$ & LUL & Adenocarcinoma & Necrosis` \\
\hline 30 & 65 & $\mathbf{F}$ & LUL & Negative & Necrosis $\dagger$ \\
\hline
\end{tabular}

«Thoracotomy showed adenocarcinoma.

$\dagger$ Thoracotomy showed aspergillosis.

$\mathrm{R}$-right; L-left; LL-lower lobe; UL-upper lobe; $\mathrm{ML}$-middle lobe; ca—carcinoma. 
$A-$ Chest radiograph $(A)$ of a 66 year old woman showing a consolidation of the anterior segment of the right upper lobe. The transbronchial lung biopsy showed chronic

inflammation. B-Wedge shaped isoechoic lesion indicating the consolidated segment, shown on the chest ultrasound scan (convex scanner).

Ultrasound guided percutaneous cutting biopsy showed tuberculosis. Arrowheads indicate the air bronchogram. Hheart; $L$-consolidated lung.

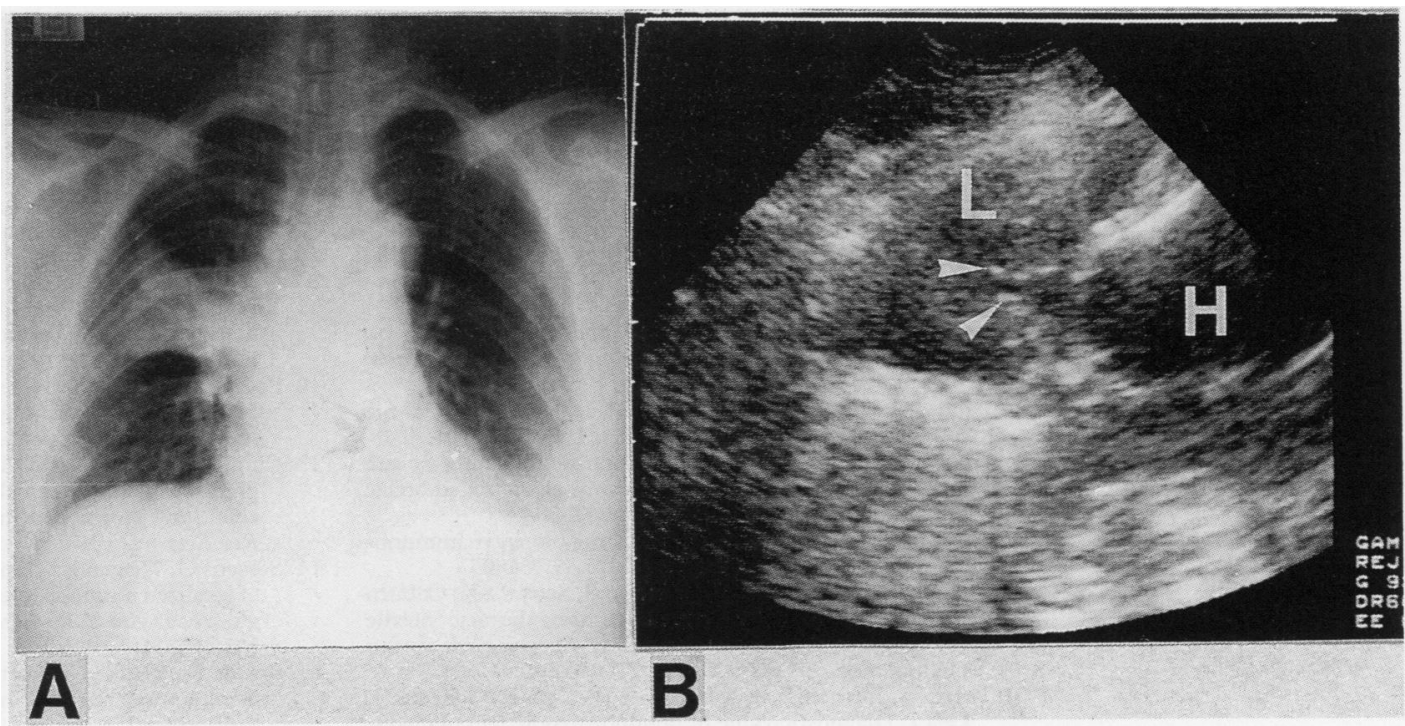

aspirations. Twenty one patients had one cutting biopsy specimen taken and nine patients two specimens because of an inadequate first specimen. The patients' characteristics and the results of the two biopsies are shown in the table. The figure illustrates a typical ultrasound image.

In nine of the 30 patients $(30 \%)$ a definite diagnosis was made from the percutaneous needle aspiration. Five patients had an infection: Aspergillus fumigatus in one, Actinobacillus actinomycetemcomitans in one, and Mycobacterium tuberculosis, grown in culture, in three.

A satisfactory specimen for histological diagnosis was obtained from 28 of the 30 patients by Trucut cutting biopsy (93\%). A definite aetiological diagnosis was made or confirmed in 17 patients $(57 \%)$. In 10 patients an infective cause was identified. Six patients had consolidation due to malignancy and one due to vasculitis. Two patients had the histological picture of acute pneumonia in the cutting biopsy specimens but no definite pathogens were identified (in one case Actinobacillus actinomycetemcomitans was cultured from the needle aspirate). Histologically, 11 patients had chronic inflammatory changes with fibrosis and had a diagnosis of chronic non-specific pneumonia. In the two remaining patients necrotic tissue only was obtained. Thoracotomy in these two patients showed an adenocarcinoma in one patient and aspergillosis in the other. Cytological examination of the needle aspirate from the former showed adenocarcinoma.

Two patients developed complications. One had a small pneumothorax and one a small haemoptysis. Both complications resolved spontaneously.

\section{Discussion}

Obtaining a definite aetiological diagnosis in cases of pulmonary consolidation may be difficult. Invasive procedures, such as fibreoptic bronchoscopy, percutaneous needle aspiration biopsy, and even open lung biopsy, may sometimes be necessary. Although flexible fibreoptic bronchoscopy with biopsy is a safe and useful procedure, the diagnostic yield is often low. ${ }^{12} 13$

Percutaneous needle aspiration provides an alternative diagnostic method for such patients. The procedure is conducted under fluoroscopic guidance. Many studies have reported its value and low complication rate ${ }^{14}$; a diagnostic yield of $31-64 \%$ has been reported..$^{2-46}$ The method is restricted by the size of the needle that can used, so that aspirated material is suitable only for cytological or microbiological examination. A large bore cutting biopsy needle provides a better tissue sample for histopathological examination and diagnostic yields of more than $70 \%$ have been reported with this method. ${ }^{15}$ The complication rate exceeds that of transbronchial lung biopsy or open lung biopsy, however, and for this reason percutaneous cutting biopsy has been abandoned by some investigators. ${ }^{16}$ The major factor contributing to the high complication rate of this method may be the image guiding system rather than the needle itself. Differentiation of vascular structures and major bronchi located in consolidated lung parenchyma may be difficult with fluoroscopic guidance, and penetration of such vessels and bronchi is presumed to be the principal cause of complications.

Recently we have shown that chest ultrasound is useful in the assessment of pulmonary consolidation. ${ }^{9}$ The major bronchi and vessels can be identified by high resolution ultrasound in conjunction with Doppler ultrasound, and the biopsy route can then be selected to avoid injury to the structures. This allows percutaneous biopsy to be conducted safely under the guidance of real time ultrasound images. In the present study percutaneous cutting biopsy was carried out safely with a high specific diagnostic yield.

We conclude that for patients with pulmonary consolidation of unknown aetiology ultrasound guided percutaneous cutting biopsy 
is a good method for providing a definite diagnosis and more invasive procedures such as open lung biopsy can be avoided. This technique may be particularly useful for immunocompromised patients with pulmonary consolidation who have failed to be diagnosed by conventional approaches.

1 Lillington GA. Lobar and segmental consolidation. In: Collins N, ed. $A$ diagnostic approach to chest diseases. Baltimore: Williams and Wilkins, 1987:173-87.

2 Palmer DL, Davidson M, Lusk R. Needle aspiration of the lung in complex pneumonias. Chest 1980;78:16-21.

3 Gherman C, Simon H. Pneumonia complicating severe underlying disease: a current appraisal of transthoracic lung puncture. Dis Chest 1965;18:297-304.

4 Greenman R, Goodall P, King D. Lung biopsy in immunocompromised hosts. Am J Med 1975;59:488-96.

5 Chaudhary S, Hughes WT, Feldman S, Sanyal SK, Coburn $\mathrm{T}$, Ossi M, et al. Percutaneous transthoracic needle aspiration of the lung. Diagnosing Pneumocystis carinit pneumonitis. Am J Dis Child 1977;131:902-7.

6 Torres A, Jiménez P, de la Bellacasa JP, Celis R, González J, Gea J. Diagnostic value of nonfluoroscopic percutaneous lung needle aspiration in patients with pneumonia. Chest 1990;98:840-4.
7 Izumi S, Tamaki S, Natori H, Kira S. Ultrasonically guided aspiration needle biopsy in disease of chest. Am Rev Respir Dis 1982;125:460-4.

8 Yang PC, Luh KT, Sheu JC, Kuo SH, Yang SP. Peripheral pulmonary lesions: ultrasonography and ultrasonically guided aspiration biopsy. Radiology 1985;155:451-6.

9 Yang PC, Luh KT, Wu HD, Chang DB, Lee LN, Kuo SH, et al. Lung tumors associated with obstructive pneumonitis: US studies. Radiology 1990;174:717-20.

10 Yang PC, Lee LN, Luh KT, Kuo SH, Yang SP. Ultrasonography of Pancoast tumor. Chest 1988;94:124-8.

11 Yu CJ, Yang PC, Chang DB, Wu HD, Lee LN, Lee YC, et al. Evaluation of ultrasonically guided biopsies of mediastinal masses. Chest 1991;100:399-405.

12 Poe RH, Utell MJ, Israel RH, Hall WJ, Eshleman JD. Sensitivity and specificity of the nonspecific transbronchial lung biopsy. Am Rev Respir Dis 1979;119:25-31.

13 Cunningham JH, Zavala DC, Corry RJ, Keim LW. Trephine air drill, bronchial brush and fiberoptic transbronchial lung biopsies in immuno-suppressed patients. Am Rev Respir Dis 1977;115:213-20.

14 Stevens $G$, Weigen J, Lillington G. Needle aspiration biopsy of localized pulmonary lesions with amplified fluoroscopic guidance. Am J Roentgenol Rad Ther Nucl Med 1968; 103:561-71.

15 Bandt P, Blank N, Castellino R. Needle diagnosis of pneumonitis: value in high risk patients. JAMA 1972; 220:1578-80.

16 Stover DE. Diagnosis of pulmonary disease in the immunocompromized host. Semin Respir Med 1989;10:89-100.

\section{BOOK NOTICES}

A Century of Tuberculosis-South African Perspectives. HM Coovadia and SR Benatar. (Pp 319; f17.50.) Cape Town: Oxford University Press, 1991. ISBN 0195705831

This South African perspective of tuberculosis over the last 100 years divides into four main sections. "History and epidemiology" covers the history of tuberculosis from early times into the prechemotherapy era, followed by the epidemiology of tuberculosis in South Africa documenting control efforts and the detailed epidemiology in various ethnic groups. "Clinical aspects" covers tuberculosis in children and adults in general, with specific chapters on pericardial, skin, neurological, and bone and joint forms, which are commonly seen in South Africa. Next the relationships between occupation and tuberculosis, particularly between mining and silicosis, are fully examined. The final section covers various aspects of diagnosis, treatment, and immunology, including the spectrum of radiographic changes; a review of the immune response to Mycobacterium tuberculosis; and diagnostic considerations in management and epidemiology. The evolution of antituberculosis chemotherapy is charted for both developed and developing countries, and it was pleasing to see the British Thoracic Society treatment guidelines listed in the milestones of tuberculosis treatment. The final section deals with the role of voluntary organisations and other aspects of tuberculosis control in South Africa. It is disappointing to see relatively little on the tuberculosis-HIV interaction, even though this will clearly be a major problem for this part of Africa. Although HIV infection is mentioned in various sections, there are no figures for the prevalence of HIVtuberculosis cases in South Africa, and nothing about local clinical experience in treatment. The book's strength is in the non-clinical sections, which clearly show the interaction of social, economical, and political factors in tuberculosis, the importance of which tends to be neglected in the developed world. In particular, the pernicious and detrimental effects of the apartheid and homeland systems on the incidence and control of tuberculosis are graphically brought out. The book will appeal to those with interests in tuberculosis and its control, but may not find its way on to departmental shelves.-LPO

Recent Advances in Respiratory Medicine 5. D M Mitchell. (Pp 312; £32.95.) Edinburgh: Churchill Livingstone, 1991. ISBN 0-433-04467-8.

Despite regular reading of the journals it is quite difficult for the practising physician to obtain a balanced view of the changes and developments occurring in all aspects of respiratory medicine over a period of time. Recent Advances in Respiratory Medicine thus has an important role. Five years seems a long time since the last volume in this series was produced, so volume 5 is very welcome. The aim, as always, is to provide up to date reviews of the growing areas in respiratory medicine, written by leaders in each subject, for consumption by doctors in training as well as the practising physician. In volume 5 , as in previous volumes, this is undoubtedly achieved. Of the 17 chapters in the book, two are devoted to asthma and four to aspects of lung transplantation, the latter being of special value for those who do not have the privilege of working in a transplant centre. Each of the other 11 chapters covers different and specific areas of respiratory medicine, ranging from cystic fibrosis to chronic obstructive lung disease. The emphasis is mainly on clinical topics but the basic sciences are not neglected, with sections of the chapters on the adult respiratory distress syndrome, asthma, cystic fibrosis, and occupational lung disease dedicated to these. Most chapters are concisely presented and extensively referenced, allowing readers to delve even further into a particular subject if they wish. It is impossible to be impressed with every aspect of any book. I would like to have seen more detail on the risk of treatment in the chapter on venous thromboembolism. The chapter on interstitial lung diseases might have been better if it had been more selective in its content rather than giving an overview of all aspects, especially when separate chapters on sarcoidosis and occupational lung disease cover some of the same ground. The price at $£ 32.95$ seems a lot for a small volume but given the amount of information crammed into the 312 pages this is perhaps unfair. These are but minor quibbles about what is an otherwise excellent volume. It should be on the bookshelf of any practising respiratory physician and should be read by all those in training in respiratory medicine. $-\mathrm{MW}$ 